\title{
The Cognitive Scale of Basic and Instrumental Activities of Daily Living for Multidomain Mild Cognitive Impairment and Dementia Patients: Validation of its Extended Version
}

\author{
Nuria Montoro-Membila1 ${ }^{1}$, Marisa Arnedo Montoro ${ }^{1,2}$, María Jesús Funes ${ }^{1,2}$ and María Rodríguez-Bailón ${ }^{3 * *}$ (i) \\ ${ }^{1}$ Mind, Brain and Behavior Research Center (CIMCYC UGR). University of Granada, Granada, Spain \\ ${ }^{2}$ Department of Experimental Psychology, University of Granada, Granada, Spain \\ ${ }^{3}$ Department of Physiotherapy (Occupational Therapy), University of Malaga, Malaga, Spain
}

(Received May 28, 2020; Final Revision April 15, 2021; Accepted April 23, 2021; First Published OnLine June 14, 2021)

\begin{abstract}
Objective: To validate an informant-based tool - the extended version of the Cognitive Scale of Basic and Instrumental Activities of Daily Living (BADL and IADL) or Ext. Cog-ADL Scale - in a larger sample and with a broader range of cognitive-functional items related to activities of daily living (ADL). Method: The Ext. Cog-ADL Scale was administered to family informants of 42 patients with dementia, 43 patients with multidomain mild cognitive impairment (mdMCI), and 23 healthy control participants. We analyzed the convergent and concurrent validity and external validity of this scale. Results: The Ext. Cog-ADL Scale demonstrated good psychometric properties. Episodic and working memory tests were the main predictors of most cognitive-functional items of the scale. While patients with dementia obtained lower scores in most error categories of the scale, affecting both BADL and IADL, mdMCI patients showed a more specific pattern of difficulties. Apart from the typical alterations in IADL, mdMCI patients also showed difficulties in several error categories related to BADL (i.e., error detection, problem solving, task self-initiation, distraction inhibition, and restore). Conclusions: The Ext. Cog-ADL Scale seems to be an adequate tool to capture the specific pattern of cognitive alterations related to IADL and BADL that differentiates dementia from mdMCI and healthy aging; it shows that mdMCI can involve specific cognitive difficulties that affect even BADL.
\end{abstract}

Keywords: Activities of daily living, Multidomain mild cognitive impairment, Dementia, Occupational therapy, Executive functions, Neuropsychology

\section{INTRODUCTION}

By definition, the diagnosis of dementia includes functional impairments (i.e., alterations in the execution of basic and instrumental activities of daily living; BADL and IADL, respectively) related to the cognitive deficits present in this patient population (Jack et al., 2018; Kamiya, Osawa, Kondo, \& Sakurai, 2018; Mograbi et al., 2018). However, the functional impact of cognitive deterioration in patients with multidomain mild cognitive impairment (mdMCI) is still under debate. In fact, mdMCI is considered a diagnostic entity in which the patient has cognitive alterations in more than one domain in the absence of dementia and with normal functioning in most activities of daily living (ADL) (Petersen, 2004). However, a large number of studies have found

*Correspondence and reprint requests to: María Rodríguez-Bailón, Department of Physiotherapy (Occupational Therapy), University of Malaga, Malaga, Spain. E-mail: mariarbailon@uma.es evidence of difficulties in IADL in this population compared to healthy aged participants (Farias et al., 2008; Jefferson et al., 2008; Kalligerou et al., 2020; Lee, Jang, \& Chang, 2019; Lindbergh, Dishman, \& Miller, 2016; Reppermund et al., 2011; Tulliani et al., 2019). In addition, the degree of functional alterations in IADL in patients with MCI is a relevant predictor of transition to dementia (Gold, 2012; Gomar et al., 2011; Luck et al., 2011; Peres et al., 2006; Tarnanas, Tsolaki, Wiederhold, Wiederhold, \& Tsolaki, 2015). Among the different types of MCI, the mdMCI subtype has been associated with higher levels of disability than the unidomain subtype in IADL tasks (Aretouli \& Brandt, 2010; Burton, Strauss, Bunce, Hunter, \& Hultsch, 2009). Therefore, it is essential to develop sensitive evaluation tools for the detection of functional problems in mdMCI. However, most functional tools (i.e., Barthel Index; Mahoney \& Barthel, 1965; Lawton \& Brody, 1969) do not make it possible to determine whether the functional limitations are 
Table 1. Example of the extended version of the Cog-ADL Scale

\begin{tabular}{|c|c|c|}
\hline \multirow{3}{*}{$\begin{array}{l}\text { Cognitive-functional } \\
\text { ability category }\end{array}$} & \multirow[b]{3}{*}{ Definitions } & Example (item) of brushing teeth \\
\hline & & $\begin{array}{l}\text { How often did he/she use to do this activity in his/ } \\
\text { her life? }\end{array}$ \\
\hline & & How often does he/she currently do this activity? \\
\hline Action schema & $\begin{array}{l}\text { Ability to complete all necessary steps of the task in } \\
\text { the correct order }\end{array}$ & $\begin{array}{l}\text { He/she knows the necessary steps to brush his/her } \\
\text { teeth and performs them in the right order (i.e., } \\
\text { putting the toothpaste on the toothbrush, brushing } \\
\text { his/her teeth and finally rinsing out). }\end{array}$ \\
\hline Error detection & Ability to detect their own errors & $\begin{array}{l}\mathrm{He} / \mathrm{she} \text { is able to notice if he/she has got toothpaste } \\
\text { on him-/herself, if he/she got wet, if he/she has put } \\
\text { too much or too little toothpaste or has not rinsed } \\
\text { out enough (i.e., ability to detect that there has } \\
\text { been an error). }\end{array}$ \\
\hline Problem solving & $\begin{array}{l}\text { Ability to solve any unexpected situation occurring } \\
\text { during the execution of an ADL }\end{array}$ & $\begin{array}{l}\mathrm{He} / \mathrm{she} \text { is able to find a solution if there is no tooth- } \\
\text { paste left, if he/she does not have a towel or if a } \\
\text { problem arises (e.g., looking for another toothpaste } \\
\text { tube). }\end{array}$ \\
\hline Task self-initiation & $\begin{array}{l}\text { Ability of the participant to self-initiate a task in an } \\
\text { autonomous manner }\end{array}$ & $\begin{array}{l}\mathrm{He} / \text { she remembers to brush when necessary (e.g., } \\
\text { after meals or before going to bed). }\end{array}$ \\
\hline Distraction inhibition & $\begin{array}{l}\text { Ability to avoid distraction and grabbing or making } \\
\text { tangential actions toward irrelevant objects which } \\
\text { were not necessary for the task at hand }\end{array}$ & $\begin{array}{l}\text { He/she gets distracted with (touches or grabs) some } \\
\text { other object in the room that is not required for } \\
\text { brushing his/her teeth. }\end{array}$ \\
\hline Object selection & $\begin{array}{l}\text { Ability to select the proper objects to perform the task } \\
\text { without replacing them with others }\end{array}$ & $\begin{array}{l}\mathrm{He} / \mathrm{she} \text { mistakes some other object for the tooth- } \\
\text { brush. }\end{array}$ \\
\hline Praxis & $\begin{array}{l}\text { Ability to make gestures and correctly handle an object } \\
\text { of the task }\end{array}$ & $\begin{array}{l}\text { He/she knows how to grab the toothbrush with his/ } \\
\text { her hand and brush, and how to put toothpaste on } \\
\text { the toothbrush. }\end{array}$ \\
\hline Restore & $\begin{array}{l}\text { Ability to reestablish everything that has been manipu- } \\
\text { lated and cleaning the work area once finished }\end{array}$ & $\begin{array}{l}\mathrm{He} / \mathrm{she} \text { puts all the utensils back and closes the tooth- } \\
\text { paste tube and the water tap. }\end{array}$ \\
\hline Semantic knowledge & Ability to recognize the use of the objects & $\begin{array}{l}\text { He/she knows what the utensils are and what they } \\
\text { are for (toothbrush, toothpaste, towel, etc.). }\end{array}$ \\
\hline
\end{tabular}

Note. ADL, activities of daily living; Cog-ADL, Cognitive Scale of Basic and Instrumental Activities of Daily Living.

caused by physical or cognitive problems. Yet, this is crucial when evaluating neurological and aged populations in which both types of difficulties may co-occur. In addition, most studies are focused on IADL, and the possibility that there might be subtle alterations present in MCI that affect even the execution of BADL remains unknown. Recently, Cornelis et al. (2017) designed an informant-based scale to assess the degree of dependency of individuals in both BADL and IADL, and also asked about the physical and/ or cognitive causes of such dependency. They found that patients with Alzheimer's disease (AD) significantly differed from healthy participants in both BADL and IADL, whereas patients with mild cognitive impairment (MCI) only showed a deterioration in IADL that was specifically caused by cognitive impairment.

In a similar vein, we designed the Preliminary Cognitive Scale of Basic and Instrumental Activities of Daily Living (Cog-ADL Scale; Rodríguez-Bailón, Montoro-Membila, García-Morán, Arnedo-Montoro, \& Funes-Molina, 2015). It is an informant-based tool whose general objective is to evaluate, in patients with mdMCI and dementia, the existence of cognitive alterations that may affect the performance of
BADL and IADL. The scale included questions about patients' abilities to follow correct action schemas as well as their skills related to error detection, problem solving, and self-initiation (see definitions in Table 1) within the context of four BADL and three IADL categories. While patients with dementia exhibited an impairment in the four cognitivefunctional BADL categories and in the three IADL categories, mdMCI patients showed an impairment in IADL. Yet, the most remarkable result was the following: by including specific cognitive items to test both types of ADL, we discovered a significant impairment of mdMCI patients in problemsolving abilities and marginally in error detection and selfinitiation categories even for BADL. These findings highlight the need for a comprehensive evaluation of the cognitive components that impact different ADL in order to better identify the subtle functional deficits in basic activities that may be present in patients with mdMCI.

\section{The Present Study}

The main aim of the present study was to develop an extended version of the preliminary informant-based Cog-ADL Scale 
Table 2. Description of the preliminary version of the Cog-ADL Scale (Preliminary-Cog-ADL Scale) and the extended version of the Cog-ADL Scale (Ext.-Cog-ADL Scale)

\begin{tabular}{|c|c|c|}
\hline & Preliminary-Cog-ADL Scale & Ext. Cog-ADL Scale \\
\hline $\begin{array}{l}\text { Number of items } \\
\text { Cognitive-functional abilities }\end{array}$ & $\begin{array}{l}28 \\
\text { Action schema } \\
\text { Error detection } \\
\text { Problem solving } \\
\text { Task self-initiation }\end{array}$ & $\begin{array}{l}69 \\
\text { Action schema } \\
\text { Error detection } \\
\text { Problem solving } \\
\text { Task self-initiation } \\
+ \text { Distraction inhibition } \\
+ \text { Object selection } \\
+ \text { Restore } \\
+ \text { Semantic knowledge } \\
+ \text { Praxis }\end{array}$ \\
\hline ADL & & \\
\hline BADL & Brushing teeth & Brushing teeth \\
\hline $\begin{array}{l}\text { Defined as "the fundamental activities to live } \\
\text { in a social world, which allow for survival } \\
\text { and well-being" (Christiansen \& Hammecker, } \\
\text { 2001, p. 156) }\end{array}$ & $\begin{array}{l}\text { Having a shower } \\
\text { Putting on makeup/shaving }\end{array}$ & $\begin{array}{l}\text { Having a shower } \\
\text { Putting on makeup/shaving }\end{array}$ \\
\hline IADL & Getting dressed & Getting dressed \\
\hline $\begin{array}{l}\text { Activities of support to daily living at home and } \\
\text { in the community that often require more } \\
\text { complex interactions compared to self-care } \\
\text { activities (American Occupational Therapy } \\
\text { Association, 2014) }\end{array}$ & $\begin{array}{l}\text { Cooking } \\
\text { Home care } \\
\text { Managing domestic finances/shopping }\end{array}$ & $\begin{array}{l}\text { Cooking } \\
\text { Home care } \\
\text { Managing domestic finances/shopping } \\
+ \text { Medication management }\end{array}$ \\
\hline
\end{tabular}

Note. ADL, activities of daily living; Cog-ADL, Cognitive Scale of Basic and Instrumental Activities of Daily Living (BADL and IADL, respectively).

by including a larger number of cognitive-functional items that may potentially impact the performance of BADL and IADL in patients with dementia or mdMCI (see the description of the previous and present versions of the scale and their differences in Table 2). The preliminary version of the CogADL Scale assesses important cognitive-functional domains in ADL. To make this scale more ecological and predictive following the performance-based literature, we included new cognitive-functional items that might impact the functional execution of these populations.

One of the new cognitive-functional items included in the extended version of the scale was distraction inhibition. It was included to identify alterations in this cognitive domain, such as the presence of tangential actions toward irrelevant objects not necessary for the task at hand (i.e., distractors). Several ADL performance-based studies have shown that this is one of the most frequent errors among patients with frontal brain damage (Niki, Maruyama, Muragaki, \& Kumada, 2009) and patients with mdMCI or dementia (RodríguezBailón et al., 2017). The second cognitive-functional item added to the new version of the scale was praxis. This item aims to isolate the presence of difficulties when using objects, such as inefficient or unnecessary gestures to manipulate a given object, even in the absence of physical deficits. This process has been explored in MCI and dementia patients with performance-based tasks by coding errors as gesture substitution, spatial misorientation, or spatial misestimation (Giovannetti et al., 2008). In fact, several studies have demonstrated the importance of assessing these skills in the diagnosis of dementia (Ahmed, Baker, Thompson, Husain, \& Butler, 2016; Benke, 1993). The third new item included was object selection and assessed the ability to select the appropriate object for a given action. Alterations in this ability have been reported in a population with AD (Giovannetti et al., 2008). An increase in this type of error in the performance of ADL has been associated with the presence of distractor objects (Cooper, Schwartz, Yule, \& Shallice, 2005) and with a deterioration in knowledge of the objects and steps of the task in patients with dementia. This could be explained by a lack of clear information to guide object selection (Roll, Giovannetti, Libon, \& Epping, 2019). The fourth item included was semantic knowledge, to analyze patients' knowledge about object identity and use. Several studies have highlighted the relationship between semantic abilities and daily functioning in populations with MCI and dementia; in fact, semantic abilities have even been found to be a predictor of daily functioning (Kirchberg et al., 2012).

Finally, we included the restore item (i.e., the ability to reestablish everything that has been manipulated and cleaning the work area once finished). This is a cognitive-functional category typically coded in ADL performance-based tests, such as the Assessment of Motor and Process Skills or AMPS (Fisher, 2010). It is also one of the processes that predict the amount of assistance that a person with $\mathrm{AD}$ requires to live independently (Liu et al., 2007). 
The present informant-based scale makes it possible to assess different cognitive-functional domains in the natural environment of the patient, where ADL are typically performed. Moreover, its administration is easier and faster than that of performance-based tasks.

Once the extended version of the Cog-ADL scale was designed, our specific aims were to test its psychometric adequacy through factor analysis as well as its convergent (i.e., functional) validity and concurrent (i.e., cognitive) validity. Finally, we aimed to test its external validity by evaluating the ability of the scale to discriminate among healthy subjects, mdMCI patients, and dementia patients in each group, both for BADL and IADL and for several cognitive-functional ability categories among them. Based on the findings from the preliminary scale and other performance-based studies, we expected to find significant differences between the dementia and control groups in all the items assessing BADL and IADL.

In addition, we expected mdMCI participants to show alterations not only in all IADL categories but also in several BADL categories, specifically in problem solving, error detection, and self-initiation.

\section{METHODS}

\section{Participants}

Forty-three patients with multidomain MCI (i.e., mdMCI group) and 42 patients with dementia (i.e., dementia group) were recruited for this study from the Dementia Outpatient Program of San Cecilio Hospital in Granada, Spain.

The mdMCI group consisted of patients in levels 2 or $3-$ compatible with MCI - of the Global Deterioration Scale or GDS (Reisberg, Ferris, de Leon, \& Crook, 1982). The dementia group consisted of 25 patients diagnosed with probable AD in levels 4 or 5 of the GDS - compatible with moderate cognitive impairment - and 17 patients with behavioral variant frontotemporal dementia (bvFTD), also in moderate stages. The diagnoses were conducted by a neurologist and a neuropsychologist specialized in dementia and MCI based on a comprehensive medical and cognitive assessment. The assessment included at least a physical examination, laboratory testing, a neuropsychological evaluation, and a brain neuroimaging study. For the diagnosis of participants with mdMCI, the following published criteria were considered (Petersen, 2004): (1) concerns of the patient or informant about the cognitive state; (2) objective evidence of impairment (greater than 1.5 SD) in more than one cognitive domain; (3) normal functioning in most ADL; and (4) absence of dementia according to the Diagnostic and Statistical Manual, 5th. Edition (DSM-5) criteria (American Psychiatric Association, 2013). Participants with dementia met the criteria of the DSM-5. The age-matched, healthy control (HC) participants $(n=23)$ were recruited from the community and did not exhibit any cognitive deficits. None of the participants of this group obtained a score lower than 28 in the MEC, the Spanish adaptation of the Mini-Mental State
Examination (Lobo et al., 1999), suggesting normal cognitive functioning (mean score $=29.63, S D=.66$ ).

Having a psychiatric illness, motor/sensory impairments or several deficits in language comprehension were exclusion criteria for all participants. Data from 58\% of the sample were taken from the preliminary version of the scale (RodríguezBailón et al., 2015). Those participants completed the new items of the extended version within two months. The Ethics Committee of the hospital authorized the research, in compliance with the Spanish legislation on the protection of personal data (Ley Orgánica de Protección de Datos de Carácter Personal 15/1999, 1999), and all participants signed an informed consent form. The study was conducted in accordance with the ethical standards of the 1964 Declaration of Helsinki.

\section{Instruments}

\section{Extended Cog-ADL Scale (Ext. Cog-ADL Scale)}

The protocol of this tool includes the following ADL: four BADL (i.e., brushing teeth, having a shower, putting on makeup/shaving, and getting dressed) and four IADL [i.e., cooking, home care (cleaning, washing up, hanging out the laundry), and managing domestic finances/shopping]. In this extended version of the scale, a new IADL was added: medication intake.

This new version of the Cog-ADL Scale assesses nine cognitive-functional ability categories, including the four categories evaluated in the preliminary version, namely (1) action schema, (2) error detection, (3) problem solving, and (4) task self-initiation, as well as five new categories described in the introduction: (5) distraction inhibition, (6) praxis, (7) object selection, (8) semantic knowledge, and (9) restore (see Table 1). The restore category was not included in the activities of getting dressed, domestic finances/shopping, or medication and the praxis category was excluded from domestic finances/shopping and medication. These exclusions were due to the fact that these items are not clearly identifiable from that cognitive perspective and their inclusion may have confused informants. As in the preliminary version of the scale, two separate items related to action schema were included in the cooking and cleaning activities, with the aim of assessing the knowledge of the sequence for both simple and complex tasks. Finally, two items were included regarding medication to address the task self-initiation category. The aim was to help the family identify potential difficulties at the beginning of the treatment and also when arranging an appointment with the doctor. A total of 69 items were included in the Ext. CogADL after adding 41 items to the preliminary scale, which consisted of 28 items (Table 2). Scores on each item and on each cognitive-functional category, since the mean of the items was calculated, ranged from 1 (lowest) to 4 (highest). Appendix 1 shows the entire tool and illustrates how these different everyday cognitive-functional items were instantiated in each ADL.

The informants (i.e., reliable people who lived with the participant) were first asked whether each ADL was part of 
the participant's present repertoire and later asked about its frequency through the questions "How often did he/she use to do this activity in his/her life?" and "How often does he/ she currently do this activity?" (response options: never, sometimes, frequently, always). Subsequently, they were instructed to answer the items about cognitive-functional abilities for each ADL that participants currently performed. The informant had to indicate at least that the patient sometimes performed the activity at present. Informants were asked to leave items unanswered if they referred to ADL previously indicated as not being part of patients' current repertoire. The frequency data on the overarching ADL categories served to ensure that these activities were currently performed by the patient, but were not analyzed.

Each cognitive-functional item had four possible response choices: never (1), sometimes (2), frequently (3), or always (4); lower scores were associated with greater impairment (scores were inverted for items that asked about limitations instead of capacities).

A blinded trained professional solved any doubts or questions of informants about what each of the items referred to.

\section{Convergent validity (i.e., functional validity)}

To assess the functional convergent validity of the scale, we obtained three traditional functional measures. The Barthel Index (Mahoney \& Barthel, 1965) was administered to determine the level of participants' functionality in BADL, and the Lawton \& Brody Scale (Lawton \& Brody, 1969) was used to evaluate IADL. In addition, a subset of the sample completed a performance-based task designed to measure ADL impairment: participants were asked to perform a task related to breakfast (Spanish ADL performance-based task; Rodríguez-Bailón et al., 2017). The design of this task was based on the Naturalistic Action Test (NAT; Schwartz, Segal, Veramonti, Ferraro, \& Buxbaum, 2002), which is a tool that makes it possible to observe different types of errors of participants during the execution of IADL. This subset of the sample was composed of 10 control participants, 11 patients with mdMCI, and 15 patients with dementia. Participants were placed in front of a table with various target and distractor objects, ensuring that they could grasp every object. They were instructed to perform an ADL: prepare a cup of coffee with milk and sugar or a toast with butter and jam. Task type was counterbalanced across participants. The performance of each participant was video recorded for later analysis, which was conducted by two independent evaluators, one of them being blinded to the patient group assignment. The types of errors made by participants were coded following the same coding system used in our prior study (see Rodríguez-Bailón et al., 2017, for a detailed description). All errors were computed together to obtain a single ADL performance-based index of functional performance and compare it with the functional measures obtained with the Ext. Cog-ADL scale. Inter-rater reliability was assessed for $20 \%$ of the sample participants, who were randomly selected.
The intraclass correlation coefficient for test reliability was above .90 in all scoring measures. The few disagreements between the coders were resolved through discussion and reassessment of the video recordings.

Based on our previous findings (Rodríguez-Bailón et al., 2015), we expected to find significant and negative correlations between participants' total number of errors in the performance-based task and their ADL scores in the extended scale.

\section{Neuropsychological screening (i.e., concurrent validity)}

To determine the cognitive concurrent validity of the everyday cognitive-functional items proposed in the scale, a standardized neuropsychological test was administered to all participants. It included an assessment of memory, executive functions, praxis, and language that lasted approximately 90 min prior to completing the scale. The Mini-Mental State Examination (Spanish adaptation: MEC; Lobo et al., 1999) was used to assess participants' overall cognitive status. Participants' short- and long-term episodic memory was assessed with the Spanish version of the Rey Auditory Verbal Learning Test (RAVLT; Miranda \& Valencia, 1997). Two measures of this test were used: number of words recalled after the first time in a free recall test and number of words recalled in the long term in a free recall test. We measured executive functions with several neuropsychological tests typically used for this purpose. More specifically, we administered the INECO Frontal Screening (SierraSanjurjo et al., 2019), which has proved to be useful to explore several types of executive functions, such as response inhibition and set shifting, abstraction, and working memory. We also used the Spanish version of Digit Span [Wechsler Adult Intelligence Scale (Third Version) or WAIS-IV] (Rosas, Tenorio, \& Pizarro, 2012) as a more specific measure of working memory. In addition, the neuropsychological protocol included an evaluation of ideomotor apraxia with the Spanish Barcelona Test (Peña-Casanova, 1990). Finally, language performance was determined with the Spanish version of the Semantic Fluency Test by asking participants to name as many animals as possible within $60 \mathrm{~s}$ (Peña-Casanova, Guardia, Bertrán-Serra, Manero, \& Jarne, 1997) Additionally, the Spanish version of the Boston Naming Test and the Boston Diagnostic Aphasia Examination Comprehension Test were administered (García-Albea, Sánchez Bernardos, \& Del Viso, 1996). The purpose of this was to facilitate the differential diagnosis and thus to rule out possible language variants of frontotemporal dementia, since having deficits in comprehension was an exclusion criterion.

\section{Procedure}

A neuropsychologist was in charge of conducting the initial neuropsychological and functional evaluation. Subsequently, a blinded trained professional explained the Ext. Cog-ADL Scale to the informant. The informant completed the scale 
while the patient was instructed on how to conduct the performance-based ADL.

\section{Data Analysis}

First, we performed an exploratory factor analysis using the eigenvalue criterion $(>1)$ to determine the number of factors extracted. We also used an oblimin rotation to verify that the ADL included in the scale were grouped into the two classic ADL categories (BADL and IADL) as in the preliminary version of the scale. To this end, we included data from the total sample of participants $(n=108)$. In this analysis, we included variables resulting from the mean of all the items in each activity of the scale. Items referring to activities which the participant had never performed in his/her life were excluded from the analysis, as well as those that he/she no longer performed.

Second, as the scores were not normally distributed, we explore the convergent (i.e., functional) validity of the scale by performing Spearman's correlations between the two main components obtained from the factor analysis (i.e., BADL and IADL) in the informant-based scale, the Barthel Index score (BADL) and the Lawton IADL Scale. We also performed Spearman's correlations between these components and the number of total errors made by the participants in the performance-based ADL task. Correlations were adjusted with the Bonferroni correction.

Third, several univariate regression analyses were conducted to analyze the concurrent (i.e., cognitive) validity between the cognitive-functional categories included in the Ext. Cog-ADL scale and the cognitive abilities measured with the neuropsychological tests described above. Each of these analyses included, as dependent variables, the average scores of all ADL for each cognitive-functional category (e.g., action schema, error detection). As predictor variables, all the analyses included the following neuropsychological measures: MEC, INECO Frontal Screening, RAVLT short-term, RAVLT longterm, Digit Span (scalar score), semantic fluency, and praxis.

Lastly, nonparametric tests (i.e., multiple between-group Kruskal-Wallis analyses and then Mann-Whitney U-Tests for two-by-two group comparisons) were conducted to determine the ability of the scale to discriminate between healthy individuals, patients with mdMCI and patients with dementia in each group, both for BADL and IADL (i.e., external validity).

The Rank-Biserial $\left(r_{B}\right)$ Correlation for nonparametric tests (Tomczak \& Tomcak, 2014) and Cohen's $d$ for parametric ones were used to calculate effect size. We separately analyzed the cognitive-functional categories of BADL and IADL (i.e., mean scores of the items).

SPSS (v.20) and JASP (v.0.8.6) statistics software was used to conduct the statistical analyses.

\section{RESULTS}

A one-way analysis of variance (ANOVA) and a chi-square test revealed that the groups did not differ in age or years of education $[F(2.105)=1.75, p=.18 ; F(2.102)=1.03$, $p=.36$, respectively] or gender $\left(\chi^{2}=.18, p=.99\right)$.
Table 3 summarizes relevant sociodemographic and clinical data. The initial stage of $\mathrm{AD}$ is frequently characterized by alterations in memory and visuoperceptive functions. By contrast, the initial stage of bvFTD is characterized by an early alteration of executive functions, with memory and visuoperceptive functions being better preserved (Sieben et al., 2012). However, as both types of dementia progress to moderate phases, the entire brain deteriorates and, consequently, the various cognitive functions are gradually impaired: $A D$ patients have deficits in executive functions and bvFTD patients have major memory problems. The absence of differences between AD and bvDFT in the above-mentioned cognitive functions evaluated in our study (Rey Auditory Verbal Learning Test - short-term $p=.152$; Rey Auditory Verbal Learning Test - long-term $p=.599$; Digit Span score $p=.810$; INECO Frontal Screening $p=.088$ ) suggests that either participants were in moderate stages of the disease or the sample was too small to detect differences between groups. Similarly, no such differences were found for the means of BADL and IADL in the Ext. Cog-ADL Scale $(p=.163$ and $p=.320$, respectively). Therefore, in order to maximize the number of participants per group for the analyses, we decided to combine participants with $\mathrm{AD}$ and those with bvDFT into a single dementia group.

\section{Factor analysis of ADL According to the Informant-Based Cog-ADL Scale}

First, we calculated the mean of all the items in each ADL. Preliminary analyses showed that the data were appropriate for factor analysis [Kaiser-Meyer-Olkin measure of sampling adequacy $=.85$; Bartlett's test of sphericity, $\chi^{2}(28)=$ $270.9, p<.001]$. The factor analysis yielded a two-component solution that accounted for $78.37 \%$ of the variance. It revealed that the items were grouped into two main categories. The means of "brushing teeth," "having a shower," "putting on makeup/shaving," and "getting dressed" loaded on the first component (with factor loadings between .93 and .81 ), which is in line with the traditional BADL category. The means of "cooking," "domestic finances/shopping," "home care," and "medication" loaded on the second component (with factor loadings ranging from .89 to .78 ), which is consistent with the traditional IADL category.

\section{Convergent (i.e., Functional) Validity: Relationship Between Everyday Cognitive-Functional Items in the Informant-Based Scale and the Functional Assessment}

The results showed a significant positive correlation between the Barthel Index and the mean of the BADL $(r=.398$; $p=.036 ; n=28)$ and also between the Barthel Index and that of the IADL ( $r=.493 ; p=.008 ; n=28)$. Likewise, there was a significant positive correlation between the Lawton-Brody Scale and the mean of the BADL $(r=.654 ; p<.001 ; n=30)$ and between this scale and the mean of the IADL $(r=.747$; $p<.001 ; n=30$ ). 
Table 3. Sociodemographic data of participants

\begin{tabular}{|c|c|c|c|c|c|c|c|c|c|}
\hline & \multirow[b]{2}{*}{$N$} & \multicolumn{2}{|c|}{ Evolution (months) } & \multicolumn{2}{|c|}{ Age (years) } & \multicolumn{2}{|c|}{ Education (years) } & \multicolumn{2}{|c|}{ Gender $(N)$} \\
\hline & & Mean & $S D$ & Mean & $S D$ & Mean & $S D$ & Men & Women \\
\hline $\mathrm{HC}$ & 23 & - & - & 69.0 & 8.3 & 8.7 & 3.3 & 10 & 13 \\
\hline mdMCI & 43 & 20.5 & 13.4 & 70.9 & 8.2 & 8.1 & 3.2 & 18 & 25 \\
\hline Dementia & 42 & 31.6 & 19.3 & 67.6 & 8.3 & 9.2 & 3.6 & 18 & 24 \\
\hline
\end{tabular}

Note. HC, healthy controls; mdMCI, multidomain mild cognitive impairment.

Table 4. Mean raw scores of the control, mdMCI, and dementia groups in the neuropsychological tests

\begin{tabular}{|c|c|c|c|c|c|c|}
\hline Test & $\mathrm{HC}$ & mdMCI & Dementia & $F(\mathrm{~d} f)$ & Group comparisons & $d$ \\
\hline MEC & $29.6(.7)$ & $24.7(4.0)$ & $21.1(5.3)$ & $30.2(2,95)$ & $\begin{array}{l}\text { HC-mdMCI** } \\
\text { mdMCI-Dementia** } \\
\text { HC-Dementia** }\end{array}$ & $\begin{array}{r}1.5 \\
.7 \\
2.2\end{array}$ \\
\hline INECO & $23.8(2.4)$ & $14.9(7.3)$ & $9.1(6.0)$ & $43.8(2,84)$ & $\begin{array}{l}\text { HC-mdMCI** } \\
\text { mdMCI-Dementia** } \\
\text { HC-Dementia** }\end{array}$ & $\begin{array}{r}1.6 \\
.9 \\
2.9\end{array}$ \\
\hline RAVLT ST & $38.4(8.2)$ & $24.2(8.7)$ & $16.9(8.7)$ & $44.2(2,96)$ & $\begin{array}{l}\text { HC-mdMCI** } \\
\text { mdMCI-Dementia** } \\
\text { HC-Dementia** }\end{array}$ & $\begin{array}{r}1.7 \\
.8 \\
2.5\end{array}$ \\
\hline RAVLT LT & $7.7(3.1)$ & $3.6(2.8)$ & $1.1(1.5)$ & $52.9(2,96)$ & $\begin{array}{l}\text { HC-mdMCI** } \\
\text { mdMCI-Dementia** } \\
\text { HC-Dementia** }\end{array}$ & $\begin{array}{l}1.4 \\
1.1 \\
3.0\end{array}$ \\
\hline Praxis & $12.0(.0)$ & $11.7(.9)$ & $9.3(3.2)$ & $17.3(2,96)$ & $\begin{array}{l}\text { HC-mdMCI } \\
\text { mdMCI-Dementia* } \\
\text { HC-Dementia* }\end{array}$ & $\begin{array}{r}.5 \\
1.2 \\
1.1\end{array}$ \\
\hline Digit Span SC & $11.2(2.0)$ & $9.3(2.6)$ & $7.1(3.4)$ & $14.3(2,84)$ & $\begin{array}{l}\text { HC-mdMCI } \\
\text { mdMCI-Dementia* } \\
\text { HC-Dementia* }\end{array}$ & $\begin{array}{r}.8 \\
.7 \\
1.4\end{array}$ \\
\hline Semantic fluency & $19.5(5.6)$ & $11.3(4.6)$ & $9.0(4.4)$ & $36.1(2,98)$ & $\begin{array}{l}\text { HC-mdMCI** } \\
\text { mdMCI-Dementia } \\
\text { HC-Dementia** }\end{array}$ & $\begin{array}{r}1.6 \\
.5 \\
2.2\end{array}$ \\
\hline
\end{tabular}

Note. HC, healthy controls; mdMCI, multidomain mild cognitive impairment; MEC, validated Spanish version of the Mini-Mental State Examination; INECO, INECO Frontal Screening; RAVLT ST, Rey Auditory Verbal Learning Test - short-term; RAVLT LT, Rey Auditory Verbal Learning Test - long-term; Digit Span SC, Digit Span Scalar Score.

$* p<.05 ; * * p<.01$.

Regarding the errors made in the performance-based task (i.e., the Spanish ADL performance-based task), there were significant negative correlations with both the mean of the BADL $(r=-.42 ; p=.010 ; n=35)$ and that of the IADL $(r=-.53 ; p=.002 ; n=32)$.

\section{Concurrent (i.e., Cognitive) Validity: Relationship Between Everyday Cognitive-Functional Items in the Informant-Based Scale and the Neuropsychological Assessment}

Regarding the neuropsychological variables, there were significant differences between the three groups studied. Performance decreased as cognitive deterioration increased and the dementia group obtained the worst results, followed by the mdMCI group (see Table 4). The mdMCI group, corroborating the diagnosis, showed cognitive alterations compared to the control group in both executive functions and memory, but not in praxis or comprehension.

We performed nine multiple regression analyses for the total sample including the following neuropsychological variables: MEC, INECO Frontal Screening, RAVLT short-term, RAVLT long-term, Digit Span (scalar score), semantic fluency, and praxis. For the analysis, we verified that there were no multicollinearity problems. Table 5 shows the results and neuropsychological tests that significantly predicted the different cognitive-functional categories of the scale. All the models significantly explained each of the cognitive-functional categories, except for the praxis category.

\section{Relationship with Clinical Diagnosis (i.e., External Validity)}

We analyzed the ability of the Ext. Cog-ADL Scale to discriminate between the three groups in the different 
Table 5. Results of a univariate regression analysis using neuropsychological factors to predict the cognitive-functional categories

\begin{tabular}{|c|c|c|c|c|c|c|c|c|c|}
\hline & $\begin{array}{l}\text { Action schema } \\
\quad(n=67)\end{array}$ & $\begin{array}{l}\text { Error detection } \\
\quad(n=60)\end{array}$ & $\begin{array}{l}\text { Problem solving } \\
\quad(n=57)\end{array}$ & $\begin{array}{l}\text { Task self- } \\
\text { initiation } \\
(n=61)\end{array}$ & $\begin{array}{l}\text { Distraction } \\
\text { inhibition } \\
(n=56)\end{array}$ & $\begin{array}{l}\text { Object selection } \\
\quad(n=58)\end{array}$ & Praxis $(n=58)$ & Restore $(n=63)$ & $\begin{array}{l}\text { S. knowledge } \\
(n=61)\end{array}$ \\
\hline & $\begin{array}{c}\mathrm{R} 2=.31 \\
F(7,59)=3.84 \\
p<.01\end{array}$ & $\begin{array}{c}\mathrm{R} 2=.42 \\
F(7,52)=5.36 \\
p<.01\end{array}$ & $\begin{array}{c}\mathrm{R} 2=.43 \\
F(7,49)=5.23 \\
p<.01\end{array}$ & $\begin{array}{c}\mathrm{R} 2=.35 \\
F(7,53)=4.1 \\
p<.01\end{array}$ & $\begin{array}{c}\mathrm{R} 2=.45 \\
F(7,48)=5.56 \\
p<.01 \\
\end{array}$ & $\begin{array}{c}\mathrm{R} 2=.31 \\
F(7,50)=3.29 \\
p<.01\end{array}$ & $\begin{array}{c}\mathrm{R} 2=.13 \\
F(7,50)=1.06 \\
p=.4\end{array}$ & $\begin{array}{c}\mathrm{R} 2=.30 \\
F(7,55)=3.42 \\
p<.01\end{array}$ & $\begin{array}{c}\mathrm{R} 2=.29 \\
F(7,53)=3.11, \\
p<.01\end{array}$ \\
\hline & $\beta$ & $\beta$ & $\beta$ & $\beta$ & $\beta$ & $\beta$ & $\beta$ & $\beta$ & $\beta$ \\
\hline MEC & .251 & .317 & .123 & .177 & .359 & -.007 & -.044 & -.123 & $.472 *$ \\
\hline INECO & $-.524 *$ & -.196 & .141 & -.235 & -.113 & .089 & -.066 & -.022 & -.126 \\
\hline RAVLT ST & -.038 & -.122 & -.243 & -.149 & .243 & $-.671^{*}$ & -.066 & -.031 & -.402 \\
\hline RAVLT LT & $.528^{*}$ & $.441^{*}$ & $.483 *$ & $.550 *$ & .028 & $.682 *$ & .186 & $.372 *$ & .313 \\
\hline Digit Span SC & $.291 *$ & .236 & $.318 *$ & $.287 *$ & .235 & $.401 *$ & .275 & $.351 *$ & .250 \\
\hline Semantic fluency & .015 & .133 & .085 & .095 & .120 & .208 & .027 & .032 & .159 \\
\hline Praxis & .127 & -.017 & -.169 & -.031 & -.169 & -.214 & .125 & .100 & -.068 \\
\hline
\end{tabular}

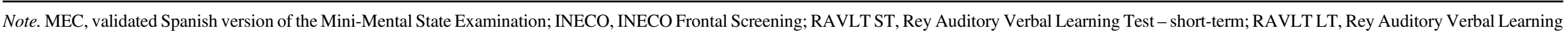
Test - long-term; Digit Span SC, Digit Span Scalar Score; S. knowledge, Semantic knowledge.

$\beta=$ standardized regression coefficient.

$* p<.05$. 
Table 6. Means and $S D$ for each cognitive-functional category and comparisons between the different groups in BADL

\begin{tabular}{|c|c|c|c|c|c|c|c|}
\hline & $\mathrm{HC}$ & mdMCI & Dementia & Comparison & $Z$ & $p$ & $r_{B}$ \\
\hline \multirow{3}{*}{ Action schema $n=108$} & \multirow{3}{*}{$4.0(.2)$} & \multirow{3}{*}{$3.8(.4)$} & \multirow{3}{*}{$3.5(.8)$} & HC-mdMCI & -1.61 & .107 & .14 \\
\hline & & & & HC-Dementia & -3.08 & $.002 *$ & .37 \\
\hline & & & & Dementia-mdMCI & -2.31 & .021 & .23 \\
\hline \multirow[t]{3}{*}{ Error detection $n=108$} & \multirow[t]{3}{*}{$3.8(.5)$} & \multirow[t]{3}{*}{$3.2(.9)$} & \multirow[t]{3}{*}{$2.7(1.0)$} & HC-mdMCI & -3.23 & $.001 *$ & .45 \\
\hline & & & & HC-Dementia & -4.82 & $.000^{*}$ & .71 \\
\hline & & & & Dementia-mdMCI & -2.11 & .034 & .26 \\
\hline \multirow[t]{3}{*}{ Problem solving $n=108$} & \multirow[t]{3}{*}{$4.0(.2)$} & \multirow[t]{3}{*}{$3.2(.9)$} & \multirow[t]{3}{*}{$2.8(1.1)$} & HC-mdMCI & -3.82 & $.000 *$ & .51 \\
\hline & & & & HC-Dementia & -5.30 & $.000 *$ & .76 \\
\hline & & & & Dementia-mdMCI & -2.22 & .026 & .27 \\
\hline \multirow{3}{*}{ Task self-initiation $n=107$} & \multirow[t]{3}{*}{$3.9(.3)$} & \multirow[t]{3}{*}{$3.4(.8)$} & \multirow[t]{3}{*}{$3.1(1.0)$} & HC-mdMCI & -3.00 & $.003 *$ & .41 \\
\hline & & & & HC-Dementia & -3.81 & $.000 *$ & .55 \\
\hline & & & & Dementia-mdMCI & -1.43 & .151 & .18 \\
\hline \multirow[t]{3}{*}{ Distraction inhibition $n=107$} & \multirow[t]{3}{*}{$3.9(.2)$} & \multirow[t]{3}{*}{$3.4(.6)$} & \multirow[t]{3}{*}{$3.1(.9)$} & HC-mdMCI & -3.77 & $.000 *$ & .49 \\
\hline & & & & HC-Dementia & -4.52 & $.000^{*}$ & .62 \\
\hline & & & & Dementia-mdMCI & -2.12 & .034 & .26 \\
\hline \multirow[t]{3}{*}{ Object selection $n=108$} & \multirow[t]{3}{*}{$4.0(.2)$} & \multirow[t]{3}{*}{$3.9(.5)$} & \multirow[t]{3}{*}{$3.6(.7)$} & HC-mdMCI & -1.34 & .180 & .11 \\
\hline & & & & HC-Dementia & -3.06 & $.002 *$ & .36 \\
\hline & & & & Dementia-mdMCI & -2.80 & $.005^{*}$ & .28 \\
\hline \multirow[t]{3}{*}{ Praxis $n=108$} & \multirow[t]{3}{*}{$4.0(.2)$} & \multirow[t]{3}{*}{$3.9(.3)$} & \multirow[t]{3}{*}{$3.7(.8)$} & HC-mdMCI & -2.19 & .028 & .23 \\
\hline & & & & HC-Dementia & -2.90 & $.004 *$ & .34 \\
\hline & & & & Dementia-mdMCI & -1.34 & .178 & .14 \\
\hline \multirow[t]{3}{*}{ Restore $n=108$} & \multirow[t]{3}{*}{$4.0(.1)$} & \multirow[t]{3}{*}{$3.5(.9)$} & $2.9(1.2)$ & HC-mdMCI & -2.51 & $.012 *$ & .27 \\
\hline & & & & HC-Dementia & -4.18 & $.000 *$ & .55 \\
\hline & & & & Dementia-mdMCI & -2.61 & $.009 *$ & .30 \\
\hline Semantic knowledge $n=108$ & $4.0(.1)$ & $3.9(.3)$ & $2.7(.5)$ & HC-mdMCI & -1.92 & .055 & .18 \\
\hline & & & & HC-Dementia & -2.67 & $.007 *$ & .30 \\
\hline & & & & Dementia-mdMCI & -1.26 & .205 & .13 \\
\hline
\end{tabular}

Note. HC, healthy controls; mdMCI, multidomain mild cognitive impairment; BADL, basic activities of daily living.

Cognitive-functional categories were based on current frequency.

*Significant data are marked with an asterisk $(*)$ after the Bonferroni correction $p<.016$.

cognitive-functional categories of the scale. We separately analyzed the differences between groups in BADL and IADL at the global level and in each cognitive category specifically.

\section{BADL}

Results showed a significant group effect in BADL $\left[\chi^{2}(2\right.$, $N=108)=35.49, p<.001]$. Specifically, the dementia group was significantly more altered than the $\mathrm{HC}$ group $(Z=5.62$, $\left.p<.001 ; r_{B}=.837\right)$ and the mdMCI group $(Z=-2.73$, $\left.p<.01 ; r_{B}=.343\right)$. More importantly, we also found that, according to informants, the mdMCI group was significantly more impaired than that of $\mathrm{HCs}(Z=-4.26, p<.001$; $\left.r_{B}=.621\right)$ in BADL. As can be seen in Table 6, comparisons between groups revealed that dementia patients showed alterations in almost every cognitive category when compared to the $\mathrm{HC}$ and mdMCI groups. By contrast, the alterations shown by the mdMCI group in BADL were specific to a set of five cognitive abilities (i.e., error detection, problem solving, task self-initiation, distraction inhibition, and restore); the differences between the mdMCI and $\mathrm{HC}$ groups in the other four categories were not significant.

\section{IADL}

Results showed a significant group effect in IADL $\left[\chi^{2}(2\right.$, $N=108)=36.13, p<.001]$. Specifically, the dementia group was significantly more altered than the $\mathrm{HC}$ group $(Z=-5.63$, $\left.p<.001 ; r_{B}=.866\right)$ and the mdMCI group $(Z=-2.69$, $\left.p<.01 ; r_{B}=.358\right)$. The mdMCI group was significantly more impaired than that of $\mathrm{HCs}\left(Z=-4.31, p<.001 ; r_{B}=.653\right)$. As can be seen in Table 7, comparisons between the different groups in each category revealed a general deterioration effect in the dementia group when compared to the HC and mdMCI groups in almost all categories. Regarding the mdMCI group, the results showed alterations in IADL in all categories when compared to the $\mathrm{HC}$ group, except for praxis and restore.

\section{DISCUSSION}

The present study establishes the extended version of the Cog-ADL Scale as a valid informant-based instrument to identify relevant cognitive alterations that may impact performance of BADL and IADL in patients with mdMCI and dementia. 
Table 7. Means and $S D$ for each cognitive-functional category and comparisons between the different groups in IADL

\begin{tabular}{|c|c|c|c|c|c|c|c|}
\hline & $\mathrm{HC}$ & mdMCI & Dementia & Comparison & $Z$ & $p$ & $r_{B}$ \\
\hline \multirow{3}{*}{ Action schema $n=91$} & \multirow{3}{*}{$3.8(.6)$} & \multirow{3}{*}{$3.5(.7)$} & \multirow{3}{*}{$2.9(1.0)$} & HC-mdMCI & -2.46 & $.014 *$ & .35 \\
\hline & & & & HC-Dementia & -3.83 & $.000 *$ & .58 \\
\hline & & & & Dementia-mdMCI & -2.45 & $.014 *$ & .34 \\
\hline \multirow[t]{3}{*}{ Error detection $n=91$} & \multirow[t]{3}{*}{$3.8(.6)$} & \multirow[t]{3}{*}{$3.1(1.0)$} & \multirow[t]{3}{*}{$2.8(.9)$} & $\mathrm{HC}-\mathrm{mdMCI}$ & -3.24 & $.001 *$ & .47 \\
\hline & & & & HC-Dementia & -4.32 & $.000 *$ & .66 \\
\hline & & & & Dementia-mdMCI & -1.46 & .144 & .20 \\
\hline \multirow[t]{3}{*}{ Problem solving $n=88$} & \multirow[t]{3}{*}{$3.9(.5)$} & \multirow[t]{3}{*}{$3.1(1.0)$} & \multirow[t]{3}{*}{$2.6(1.1)$} & HC-mdMCI & -3.89 & $.000 *$ & .55 \\
\hline & & & & HC-Dementia & -4.34 & $.000 *$ & .64 \\
\hline & & & & Dementia-mdMCI & -1.62 & .106 & .23 \\
\hline \multirow[t]{3}{*}{ Task self-initiation $n=93$} & \multirow[t]{3}{*}{$3.9(.5)$} & \multirow[t]{3}{*}{$3.3(.8)$} & \multirow[t]{3}{*}{$2.8(1.0)$} & HC-mdMCI & -3.48 & $.001 *$ & .47 \\
\hline & & & & HC-Dementia & -4.55 & $.000 *$ & .67 \\
\hline & & & & Dementia-mdMCI & -2.46 & $.014 *$ & .34 \\
\hline \multirow[t]{3}{*}{ Distraction inhibition $n=89$} & \multirow[t]{3}{*}{$3.9(.3)$} & \multirow[t]{3}{*}{$3.4(.8)$} & \multirow[t]{3}{*}{$3.1(.9)$} & HC-mdMCI & -2.96 & $.003 *$ & .38 \\
\hline & & & & HC-Dementia & -3.89 & $.000 *$ & .55 \\
\hline & & & & Dementia-mdMCI & -1.69 & .091 & .23 \\
\hline \multirow[t]{3}{*}{ Object selection $n=90$} & \multirow[t]{3}{*}{$4.0(.0)$} & \multirow[t]{3}{*}{$3.7(.7)$} & \multirow[t]{3}{*}{$3.4(1.0)$} & HC-mdMCI & -2.69 & $.007 *$ & $-^{\mathrm{a}}$ \\
\hline & & & & HC-Dementia & -3.20 & $.001 *$ & $-^{\mathrm{a}}$ \\
\hline & & & & Dementia-mdMCI & -.99 & .326 & .12 \\
\hline \multirow[t]{3}{*}{ Praxis $n=90$} & \multirow[t]{3}{*}{$3.9(.3)$} & \multirow[t]{3}{*}{$3.8(.6)$} & \multirow[t]{3}{*}{$3.7(.7)$} & HC-mdMCI & -1.33 & .181 & .11 \\
\hline & & & & HC-Dementia & -1.84 & .065 & .19 \\
\hline & & & & Dementia-mdMCI & -.70 & .487 & .07 \\
\hline \multirow[t]{3}{*}{ Restore $n=92$} & \multirow[t]{3}{*}{$3.7(.8)$} & \multirow[t]{3}{*}{$3.4(1.0)$} & $2.8(1.2)$ & HC-mdMCI & -1.27 & .204 & .16 \\
\hline & & & & HC-Dementia & -3.07 & $.002 *$ & .45 \\
\hline & & & & Dementia-mdMCI & -2.28 & .023 & .30 \\
\hline Semantic knowledge $n=108$ & $3.9(.4)$ & $3.2(1.3)$ & $2.7(1.6)$ & HC-mdMCI & -3.32 & $.001 *$ & .43 \\
\hline & & & & HC-Dementia & -3.79 & $.000 *$ & .51 \\
\hline & & & & Dementia-mdMCI & -1.09 & .276 & .13 \\
\hline
\end{tabular}

Note. HC, healthy controls; mdMCI, multidomain mild cognitive impairment; IADL, instrumental activities of daily living.

Cognitive-functional categories were based on current frequency.

* Significant data are marked with an asterisk $(*)$ after the Bonferroni correction $p<.016$.

$-^{\mathrm{a}}=$ Variance $=0$ in object selection after grouping in group ( $\mathrm{HC}$ and $\left.\mathrm{mdMCI}\right)$

The extended version of the Cog-ADL Scale provided additional evidence of a hierarchy in cognitive-functional decline, with dementia altering functionality in most BADL and IADL and most of the cognitive processes underlying them. This is consistent with prior studies aimed at testing the cognitive alterations that affect BADL and IADL in this population (Cornelis et al., 2017; Farias et al., 2008; Hindmarch, Lehfeld, de Jongh, \& Erzigkeit, 1998; Rodríguez-Bailón et al., 2015).

By contrast, the mdMCI group showed an intermediate pattern, with greater cognitive and functional alterations than those of the healthy aging group, although significantly lower than those of the dementia group. The results obtained with this new version of the scale expand those reported with the preliminary Cog-ADL Scale (Rodríguez-Bailón et al., 2015). The addition of new cognitive-functional items for each ADL category in the extended version of the Cog-ADL Scale revealed a larger pattern of alterations not only in IADL but also in BADL. The informants perceived deficits in their relatives with mdMCI in all categories of IADL except for manipulation abilities and restore. Surprisingly, those informants also detected errors in the performance of BADL that affected five cognitive-functional categories: problem solving, error detection, task self-initiation, distraction inhibition, and restore. However, another group of cognitive abilities such as action schema, object selection, praxis, and semantic knowledge were either not altered at all or only altered in more complex IADL in the mdMCI group compared the group of HCs. Categories that were affected in BADL were related to a "higher level" of attentional control necessary in situations that depend on changes in the circumstances of the task. In such situations, circumstances may change from one episode to another, thus requiring larger doses of flexible thinking, working memory, or long-term memory recovery (e.g., remembering how we dealt with an empty toothpaste container situation in the past). There is substantial evidence in the literature showing that such "higher level" cognitive alterations are lower in the MCI population than in the healthy elder population (e.g., flexible thinking studies: Fusi et al., 2020; working memory and controlling distraction: Deiber et al., 2011; Kochan et al., 2011; Missonnier et al., 2007; Saunders \& Summers, 2010; inhibition tasks: Clément et al., 2012; Sinai et al., 2010; long-term memory: Rodríguez-Bailón et al., 2017).

By contrast, no alterations were observed in situations that depended on the task schedule or the semantic representation 
of the objects that composed them, which may involve a much more automatic activation (Norman \& Shallice, 1983).

We believe that, with the inclusion of examples in the scale, we may have increased the informants' ability to dissociate between the different cognitive alterations that can affect each ADL, which could explain the ADL process dissociation described above. Although more research is still needed to disentangle such controversy among studies, the present results may have direct implications for future revisions of the diagnostic criteria for $\mathrm{mdMCI}$, as they are often exclusively described in terms of alterations in IADL.

Nevertheless, the finding of cognitive alterations in BADL in mdMCI patients is not consistent with prior studies in which cognitive alterations in MCI were restricted to IADL (Cornelis et al., 2017). A potential explanation of such discrepancy may be related to the fact that, in those studies, informants were asked to consider the degrees of cognitive dependency in broader terms (i.e., whether such dependency was due to cognitive or physical causes or both); in the present scale, however, we asked informants to distinguish between very specific cognitive abilities and provided examples of each ability.

Regarding the psychometric properties of the extended version of the Cog-ADL Scale, we found evidence of high internal consistency of the items that measured BADL and of those that measured IADL. We also found high convergent (i.e., functional) validity, which was explored with a performance-based ADL task (Spanish ADL performance-based task) and questionnaires of general functionality (i.e., Barthel Index and Lawton \& Brody Scale).

Multiple regression analyses explored the concurrent validity between the neuropsychological test scores and the cognitive-functional categories in the results of the whole sample (i.e., patients with dementia, patients with mdMCI, and HC participants). All models were significant, except the praxis cognitive-functional category. We expected to find a relationship between this cognitive-functional category and the neuropsychological measure of praxis. The absence of a significant correlation may be due to the fact that this neuropsychological test asks participants to merely make artificial gestures with their hands, which is far from the practical requirements to use objects in everyday life.

The most predictive tests for functional-cognitive categories of the Ext. Cog-ADL Scale were measures of general cognitive function (MEC), memory, and executive functions. These instruments predicted the functioning of most (i.e., seven out of nine) of the everyday cognitive categories of the scale: action schema, error detection, task self-initiation, problem solving, object selection, restore, and semantic knowledge. This pattern of results is consistent with previous research and the preliminary version of this scale (BellMcGinty, Podell, Franzen, Baird, \& Williams, 2002; Boyle, Paul, Moser, \& Cohen, 2004; Cahn-Weiner, Boyle, \& Malloy, 2002; Farias et al., 2008; Goldstein, McCue, Rogers, \& Nussbaum, 1992; Mccue, Rogers, \& Goldstein, 1990; Richardson, Nadler, \& Malloy, 1995;
Seligman, Giovannetti, Sestito, \& Libon, 2013; Sikkes \& Rotrou, 2014).

Although the model was predictive, none of the tests explained item distraction inhibition in a substantial way. As stated in the introduction, many performance-based studies have found this type of cognitive error to be a common occurrence associated with deficits in executive functions in this population. Future studies including more specific measures of executive functions, such as selective attention, may help determine the nature of this cognitive-functional category.

The current study has limitations. Although the sample was larger than the one tested in the preliminary version of the scale, this increase might be insufficient. A larger sample with new participants would be required to establish this scale as a diagnostic test and to confirm that the different items are assigned to the expected cognitive-functional categories.

Likewise, given the small sample, patients with $\mathrm{AD}$ and bvFTD were included in a single dementia group. Future research including a larger sample of patients with different cognitive profiles might help further test whether the present ADL scale is able to identify different cognitive-functional profiles among this population and, therefore, to help guide more specific interventions.

In conclusion, despite its limitations, the present study adds to the accumulating evidence of everyday cognitive difficulties in patients with mdMCI and dementia. Our findings suggest that, according to the information provided by their relatives, these patients show clear alterations not only in IADL but also in several cognitive abilities involved in BADL, such as error detection, problem solving, distraction inhibition, self-initiation, and restore. These findings differ from what is established by the diagnostic criteria for mdMCI. The present results, together with those of previous studies (Kaur, Belchior, Gelinas, \& Bier, 2016; Mis, Devlin, Drabick, \& Giovannetti, 2019; Rodríguez-Bailón et al., 2015, 2017), show the need to pay special attention to the evaluation of subtle cognitive processes involved in everyday tasks. The contribution of our study to the existing knowledge about the cognitive-functional deficits that characterize patients with mdMCI and dementia in initial stages can help in the early diagnosis of this population using a quick and simple tool that can assess patients in their everyday contexts. It can also improve the therapeutic approach, guiding the early delivery of various interventions based on the different patterns of cognitive-functional errors, which could help delay the degenerative process.

\section{SUPPLEMENTARY MATERIAL}

To view supplementary material for this article, please visit https://doi.org/10.1017/S1355617721000758

\section{ACKNOWLEDGMENTS}

We thank Francisco Javier Baron López for his collaboration. We also thank the participants and their family members for their cooperation. 


\section{FINANCIAL SUPPORT}

This research was supported by the Spanish Ministry of Science and Innovation through a $\mathrm{PhD}$ research fellowship awarded to the last author (FPI BES-2009-020741), the Regional Government of Andalusia, Spain, through a research project granted to the second and last authors (Junta de Andalucía SEJ-6351) and the Fundación para la Investigación Biosanitaria de Andalucía Oriental Alejandro Otero, a foundation for biomedical research in Eastern Andalusia, through a scholarship granted to the first author. Funding for open access charge: University of Málaga.

\section{CONFLICT OF INTEREST}

The authors have nothing to disclose.

\section{REFERENCES}

Ahmed, S., Baker, I., Thompson, S., Husain, M., \& Butler, C.R. (2016). Utility of testing for apraxia and associated features in dementia. Journal of Neurology, Neurosurgery \& Psychiatry, 87(11), 1158-1162. doi: 10.1136/jnnp-2015-312945

American Occupational Therapy Association (2014). Occupational therapy practice framework: domain and process (3rd edition). American Journal of Occupational Therapy, 68(1), 1-48. doi: 10.5014/ajot.2014.682006

American Psychiatric Association (2013). Diagnostic and Statistical Manual of Mental Disorders (5th ed.). Washington, DC: Author.

Aretouli, E., \& Brandt, J. (2010). Everyday functioning in mild cognitive impairment and its relationship with executive cognition. International Journal of Geriatric Psychiatry, 25(3), 224-233. doi: 10.1002/gps.2325

Bell-McGinty, S., Podell, K., Franzen, M., Baird, A.D., \& Williams, M.J. (2002). Standard measures of executive function in predicting instrumental activities of daily living in older adults. International Journal of Geriatric Psychiatry, 17(9), 828-834. doi: $10.1002 /$ gps.646

Benke, T. (1993). Two forms of apraxia in Alzheimer's disease. Cortex, 29(4), 715-725. doi: 10.1016/s0010-9452(13)80292-4

Boyle, P.A., Paul, R.H., Moser, D.J., \& Cohen, R.A. (2004). Executive impairments predict functional declines in vascular dementia. Clinical Neuropsychologist, 18(1), 75-82. doi: 10. 1080/13854040490507172

Burton, C.L., Strauss, E., Bunce, D., Hunter, M.A., \& Hultsch, D.F. (2009). Functional abilities in older adults with mild cognitive impairment. Gerontology, 55(5), 570-581. doi: 10.1159/000228918

Cahn-Weiner, D., Boyle, P.A., \& Malloy, P.F. (2002). Tests of executive function predict instrumental activities of daily living in community-dwelling older individuals. Applied Neuropsychology, 9(3), 187-191. doi: 10.1207/s15324826an0903_8

Christiansen, C.H., \& Hammecker, C.L. (2001). Self care. In B.R. Bonder \& M.B. Wagner (Eds.), Functional Performance in Older Adults (pp. 155-175). Philadelphia: F. A. Davis.

Clément, F., Gauthier, S., \& Belleville, S. (2012). Executive functions in mild cognitive impairment: emergence and breakdown of neural plasticity. Cortex, 49, 1268-1279. doi: 10.1016/j.cortex. 2012.06.004
Cooper, R., Schwartz, M., Yule, P., \& Shallice, T. (2005). The simulation of action disorganisation in complex activities of daily living. Cognitive Neuropsychology, 22, 959-1004. doi: 10.1080/ 02643290442000419

Cornelis, E., Gorus, E., Beyer, I., Bautmans, I., \& De Vriendt, P. (2017). Early diagnosis of mild cognitive impairment and mild dementia through basic and instrumental activities of daily living: development of a new evaluation tool. PLoS Medicine, 14(3), e1002250. doi: 10.1371/journal.pmed.1002250

Deiber, M.P., Ibáñez, V., Herrmann, F., Rodríguez, C., Emch, J., Missonnier, P., ... Giannakopoulos, P. (2011). Face short-term memory-related electroencephalographic patterns can differentiate multi- versus single-domain amnestic mild cognitive impairment. Journal of Alzheimer Disease, 26, 157-169. doi: 10.3233/ JAD-2011-110170

Farias, S.T., Mungas, D., Cahn-Weiner, D., Baynes, K., Reed, B.R., Jagust, W., \& DeCarli, C. (2008). The measurement of Everyday Cognition (ECog): scale development and psychometric properties. Neuropsychology, 22(4), 531-544. doi: 10.1037/0894-4105. 22.4.531

Fisher, A. (2010). Assessment of Motor and Process Skills: Vol 2. User manual (7th ed.). Fort Collins, CO: Three Star Press.

Fusi, G., Ferrari, E., Zanetti, M., Crepaldi, M., Bersanini, C., Paladino, A., ... Rusconi, M.L. (2020). A comparison of divergent thinking abilities between healthy elderly subjects and MCI patients: preliminary findings and implications. Frontiers in Psychology, 11. doi: 10.3389/fpsyg.2020.00738

García-Albea, J.E., Sánchez Bernardos, M.L., \& Del Viso, S. (1996). Test de Boston para el diagnóstico de la afasia: adaptación española. Madrid: Editorial Medica Panamericana.

Giovannetti, T., Bettcher, B.M., Brennan, L., Libon, D.J., Kessler, R.K., \& Duey, K. (2008). Coffee with jelly or unbuttered toast: commissions and omissions are dissociable aspects of everyday action impairment in Alzheimer's disease. Neuropsychology, 224(2), 235-245.

Gold, D.A. (2012). An examination of instrumental activities of daily living assessment in older adults and mild cognitive impairment. Journal of Clinical and Experimental Neuropsychology, 34(1), 11-34. doi: 10.1080/13803395.2011.614598

Goldstein, G., McCue, M., Rogers, J., \& Nussbaum, P.D. (1992). Diagnostic differences in memory test based predictions of functional capacity in the elderly. Neuropsychological Rehabilitation, 2(4), 307-317. doi: 10.1080/09602019208401416

Gomar, J.J., Bobes-Bascaran, M.T., Conejero-Goldberg, C., Davies, P., Goldberg, T.E., \& Alzheimer's Disease Neuroimaging Initiative (2011). Utility of combinations of biomarkers, cognitive markers, and risk factors to predict conversion from mild cognitive impairment to Alzheimer disease in patients in the Alzheimer's disease neuroimaging initiative. Archives of General Psychiatry, 68(9), 961-969. doi: 10.1001/ archgenpsychiatry.2011.96

Hindmarch, I., Lehfeld, H., de Jongh, P., \& Erzigkeit, H. (1998). The Bayer activities of daily living scale (B-ABL). Dementia and Geriatric Cognitive Disorders, 9, 20-26. doi: 10.1159/000051195

Jack, C.R., Bennett, D.A., Blennow, K., Carrillo, M.C., Dunn, B., Haeberlein, S.B., ... Sperling, R. (Contributors) (2018). NIAAA Research Framework: toward a biological definition of Alzheimer's disease. Alzheimer's \& Dementia, 14(4), 535-562. doi: 10.1016/j.jalz.2018.02.018

Jefferson, A.L., Byerly, L.K., Vanderhill, S., Lambe, S., Wong, S., Ozonoff, A., \& Karlawish, J.H. (2008). Characterization of activities of daily living in individuals with mild cognitive impairment. 
American Journal of Geriatric Psychiatry, 16(5), 375-383. doi: 10.1097/JGP.0b013e318162f197

Kalligerou, F., Fieo, R., Paraskevas, G.P., Zalonis, I., Kosmidis, M.H., Yannakoulia, M., \& Scarmeas, N. (2020). Assessing functional status using the IADL-extended scale: results from the HELIAD study. International Psychogeriatrics, 32(9), 1045-1053. doi: 10.1017/s1041610219001091

Kamiya, M., Osawa, A., Kondo, I., \& Sakurai, T. (2018). Factors associated with cognitive function that cause a decline in the level of activities of daily living in Alzheimer's disease. Geriatrics \& Gerontology International, 18(1), 50-56. doi: 10.1111/ggi.13135

Kaur, N., Belchior, P., Gelinas, I., \& Bier, N. (2016). Critical appraisal of questionnaires to assess functional impairment in individuals with mild cognitive impairment. International Psychogeriatrics, 28(9), 1425-1439. doi: 10.1017/ S104161021600017X

Kirchberg, B.C., Cohen, J.R., Adelsky, M.B., Buthorn, J.J., Gomar, J., Gordon, M., \& Goldberg, T.E. (2012). Semantic distance abnormalities in mild cognitive impairment: their nature and relationship to function. The American Journal of Psychiatry, 169(12), 1275-1283.

Kochan, N.A., Breakspear, M., Valenzuela, M., Slavin, M.J., Brodaty, H., Wen, W., ... Sachdev, P.S. (2011). Cortical responses to graded working memory challenge predict functional decline in mild cognitive impairment. Biological Psychiatry, 70(2), 123-130. doi: 10.1016/j.biopsych.2011.03.006

Lawton, M., \& Brody, E. (1969). Assessment of older people-selfmaintaining and instrumental activities of daily living. Gerontologist, 9(3P1), 179.

Lee, M.T., Jang, Y., \& Chang, W.Y. (2019). How do impairments in cognitive functions affect activities of daily living functions in older adults? PLoS One, 14(6), e0218112. doi: 10.1371/ journal.pone.0218112

Lindbergh, C.A., Dishman, R.K., \& Miller, L.S. (2016). Functional disability in mild cognitive impairment: a systematic review and meta-analysis. Neuropsychology Review, 26(2), 129-159. doi: 10.1007/s11065-016-9321-5

Liu, K.P.Y., Chan, C.C.H., Chu, M.M.L., Ng, T.Y.L., Chu, L.W., Hui, F.S.L., ... Fisher, A.G. (2007). Activities of daily living performance in dementia. Acta Neurologica Scandinavica, 116(2), 91-95. doi: 10.1111/j.1600-0404.2007.00800.x

Lobo, A., Saz, P., Marcos, G., Día, J.L., de la Cámara, C., Ventura, T., ... Aznar, S. (1999). Revalidation and standardization of the cognition mini-exam (first Spanish version of the MiniMental Status Examination) in the general geriatric population. Medicina Clinica, 112(20), 767-774.

Luck, T., Luppa, M., Angermeyer, M.C., Villringer, A., König, H.H., \& Riedel-Heller, S.G. (2011). Impact of impairment in instrumental activities of daily living and mild cognitive impairment on time to incident dementia: results of the Leipzig Longitudinal Study of the Aged. Psychological Medicine, 41(5), 1087-1097. doi: 10.1017/S003329171000142X

Mahoney, F.I., \& Barthel, D.W. (1965). Functional evaluation: the Barthel Index. Maryland State Medical Journal, 14, 56-61.

Mccue, M., Rogers, J., \& Goldstein, G. (1990). Relationships between neuropsychological and functional assessment in elderly neuropsychiatric patients. Rehabilitation Psychology, 35(2), 91-99.

Miranda, J.P., \& Valencia, R.R. (1997). English and Spanish versions of a memory test: word length effects versus spoken duration effects. Hispanic Journal of Behavioral Sciences, 19(2), 171-181.

Mis, R., Devlin, K., Drabick, D., \& Giovannetti, T. (2019). Heterogeneity of informant-reported functional performance in mild cognitive impairment: a latent profile analysis of the functional activities questionnaire. Journal of Alzheimer's Disease, 68(4), 1611-1624. doi: 10.3233/JAD-180975

Missonnier, P., Deiber, M.P., Gold, G., Herrmann, F.R., Millet, P., Michon, A., ... Giannakopoulos, P. (2007). Working memory load-related electroencephalographic parameters can differentiate progressive from stable mild cognitive impairment. Neuroscience, 150(2), 346-356. doi: 10.1016/j.neuroscience. 2007.09.009

Mograbi, D.C., Morris, R.G., Fichman, H.C., Faria, C.A., Sanchez, M.A., Ribeiro, P.C.C., \& Lourenço, R.A. (2018). The impact of dementia, depression and awareness on activities of daily living in a sample from a middle-income country. International Journal of Geriatric Psychiatry, 33(6), 807-813. doi: 10.1002/gps.4765

Niki, C., Maruyama, T., Muragaki, Y., \& Kumada, T. (2009). Disinhibition of sequential actions following right frontal lobe damage. Cognitive Neuropsychology, 26(3), 266-285. doi: 10. 1080/02643290903028484

Norman, D.A., \& Shallice, T. (1983). Attention to action. Willed and automatic control of behavior. Bulletin of the Psychonomic Society, 21(5), 354-354.

Peña-Casanova, J. (1990). Programa integrado de exploración neuropsicológica. Test Barcelona. Barcelona: Masson.

Peña-Casanova, J., Guardia, J., Bertrán-Serra, I., Manero, R.M., \& Jarne, A. (1997). Versión abreviada del test Barcelona (I): subtests y perfiles normales. Neurología, 12, 99-111.

Peres, K., Chrysostome, V., Fabrigoule, C., Orgogozo, J.M., Dartigues, J.F., \& Barberger-Gateau, P. (2006). Restriction in complex activities of daily living in MCI: impact on outcome. Neurology, 67(3), 461-466. doi: 10.1212/01.wnl.0000228228. 70065.f1

Petersen, R.C. (2004). Mild cognitive impairment as a diagnostic entity. Journal of Internal Medicine, 256(3), 183-194. doi: 10. 1111/j.1365-2796.2004.01388.x

Reisberg, B., Ferris, S.H., de Leon, M.J., \& Crook, T. (1982). The Global Deterioration Scale for assessment of primary degenerative dementia. The American Journal of Psychiatry, 139(9), 1136-1139. doi: 10.1176/ajp.139.9.1136

Reppermund, S., Sachdev, P.S., Crawford, J., Kochan, N.A., Slavin, M.J., Kang, K., Brodaty, H. (2011). The relationship of neuropsychological function to instrumental activities of daily living in mild cognitive impairment. International Journal of Geriatric Psychiatry, 26(8), 843-852. doi: 10.1002/gps.2612

Richardson, E.D., Nadler, J.D., \& Malloy, P.F. (1995). Neuropsychologic prediction of performance measures of daily living skills in geriatric patients. Neuropsychology, 9(4), 565-572. doi: 10.1037/0894-4105.9.4.565

Rodríguez-Bailon, M., Montoro-Membila, N., Garcia-Morán, T., Luisa Arnedo-Montoro, M., \& Funes Molina, M.J. (2015). Preliminary cognitive scale of basic and instrumental activities of daily living for dementia and mild cognitive impairment. Journal of Clinical and Experimental Neuropsychology, 37(4), 339-353. doi: 10.1080/13803395.2015.1013022

Rodríguez-Bailón, M., García-Morán, T., Montoro-Membila, N., Ródenas-García, E., Montoro, M.A., \& Funes Molina, M.J. (2017). Positive and negative consequences of making coffee among breakfast related irrelevant objects: evidence from MCI, 
dementia, and healthy ageing. Journal of the International Neuropsychological Society, 23(6), 481-492. doi: 10.1017/ S135561771700025X

Roll, E.E., Giovannetti, T., Libon, D.J., \& Eppig, J. (2019). Everyday task knowledge and everyday function in dementia. Journal of Neuropsychology, 13(1), 96-120. doi: 10.1111/jnp.12135

Rosas, R., Tenorio, D., \& Pizarro, M.M. (2012). WAIS-IV: Manual de administración y corrección:versión estandarizada en Chile. Santiago, Chile: NCS Pearson.

Saunders, N.L., \& Summers, M.J. (2010). Attention and working memory deficits in mild cognitive impairment. Journal of Clinical and Experimental Neuropsychology, 32(4), 350-357. doi: 10.1080/13803390903042379

Schwartz, M.F., Segal, M., Veramonti, T., Ferraro, M., \& Buxbaum, L.J. (2002). The Naturalistic Action Test: a standardized assessment for everyday action impairment. Neuropsychological Rehabilitation, 12(4), 311-339. doi: 10. 1080/09602010244000084

Seligman, S.C., Giovannetti, T., Sestito, J., \& Libon, D.J. (2013). A new approach to the characterization of subtle errors in everyday action: implications for mild cognitive impairment. Clinical Neuropsychologist, 28(1), 97-115. doi: 10.1080/13854046. 2013.852624

Sieben, A., Van Langenhove, T., Engelborghs, S., Martin, J.-J., Boon, P., Cras, P., De Deyn, P.-P., Santens, P., Van Broeckhoven, C., \& Cruts, M. (2012). The genetics and neuropathology of frontotemporal lobar degeneration. Acta Neuropathologica, 124(3), 353-372. doi: $10.1007 / \mathrm{s} 00401-012-1029-\mathrm{x}$
Sierra Sanjurjo, N., Saraniti, A.B., Gleichgerrcht, E., Roca, M., Manes, F., \& Torralva, T. (2019). The IFS (INECO Frontal Screening) and level of education: normative data. Applied Neuropsychology: Adult, 26(4), 331-339. doi: 10.1080/ 23279095.2018.1427096

Sikkes, S.A.M., \& Rotrou, J. de (2014). A qualitative review of instrumental activities of daily living in dementia: what's cooking? Neurodegenerative Disease Management, 4(5), 393-400. doi: $10.2217 / \mathrm{nmt} .14 .24$

Sinai, M., Phillips, N.A., Chertkow, H., \& Kabani, N.J. (2010). Task switching performance reveals heterogeneity amongst patients with mild cognitive impairment. Neuropsychology, 24(6), 757-774. doi: 10.1037/a0020314.

Tarnanas, I., Tsolaki, A., Wiederhold, M., Wiederhold, B., \& Tsolaki, M. (2015). Five-year biomarker progression variability for Alzheimer's disease dementia prediction: can a complex instrumental activities of daily living marker fill in the gaps? Alzheimer's \& Dementia: Diagnosis, Assessment \& Disease Monitoring, 1(4), 521-532. doi: 10.1016/j.dadm.2015.10.005

Tomczak, M. \& Tomcak, E. (2014). The need to report effect size estimates revisited. An overview of some recommended measures of effect size. Trends Sport Sciences, 1(21), 19-25.

Tulliani, N., Bissett, M., Bye, R., Chaudhary, K., Fahey, P., \& Liu, K.M.Y. (2019). The efficacy of cognitive interventions on the performance of instrumental activities of daily living in individuals with mild cognitive impairment or mild dementia: protocol for a systematic review and metaanalysis. Systematic Reviews, 8(222). doi: 10.1186/s13643-019-1135-0 